\title{
Fretting wear under magnetic field
}

\author{
Y. Nasu $^{1} \&$ K. Sato ${ }^{2}$ \\ ${ }^{1}$ Oyama National College of Technology, Japan \\ ${ }^{2}$ Graduate School of Engineering, Chiba University, Japan
}

\begin{abstract}
In our current society, based on science and technology, many kinds of electrical and mechanical systems are working under magnetic fields which become of a higher level year-over-year. The materials used in the above mentioned systems have suffered exposure to undefined risks due to magnetic effects. The authors have studied the complicated effect of magnetic fields on fretting wear, e.g. in some cases the effect is to accelerate wear volume and in other cases it does not. In the present paper the authors describe the outline of the effect of magnetic fields on fretting wear and the state of the art.
\end{abstract}

Keywords: magnetic field, fretting wear, friction and wear, material.

\section{Introduction}

Electrical and mechanical systems, such as electric generators, magnetic readers, linier motors, medical devices, and so on are working under the artificial magnetic field environment. According to recent progress in science and technology, devices and systems are increasingly exposed to and operate under higher magnetic fields year on year. The materials composed of the devices and systems, therefore, are exposed to an undefined risk situation, because the effects of magnetic fields have not been obviously examined. The problem is that the effects are different from conditions due to material, intensity of magnetic field, and working conditions of stress and vibration, and so on.

The wear of machine elements, or the contact surfaces, between them, is a reason for defining the lifetime of the electric apparatuses, such as conveyance equipment, rotating machinery, and precision electronics [1]. The wear of machine elements may be related to the intensity of the magnetic field and their lifetime is able to be controlled by changing the magnetic field. A number of practical examinations are, however, necessary for doing a good quality jobs in 
design and maintenance. This is the motivation for our research through experiments to examine the effects of magnetic fields on fretting wear which is an especially important job in design and maintenance.

In the present paper, the effect of magnetic fields on wear and on friction aspects in the materials of the systems and devices has been reviewed. In addition, the result that the authors have studied of the effect of magnetic fields on fretting wear has been described. Furthermore, the relationship between the magnetic fields environment and advances based on science and technology has been surveyed.

\section{Failure of urban systems under magnetic fields}

Engineering structures and machines are frequently subjected to attacks due to corrosion, fatigue, and wear during working. As it is reported that the magnetic field affects these failures, deflection of the corrosion pattern [2] and improvement of the fatigue life [3], the authors summarize and focus on the effect of magnetic fields in wear as below.

\subsection{Effects of magnetic field on wear process}

Metal wear and wear process are affected by the kind and type of, direction of, and level of magnetic field, material, atmosphere, time, frequency, and so on.

\subsubsection{Wear process under magnetic field}

Muju and Ghosh [4], who firstly studied the effects of magnetic fields on wear, reported that magnetic fields affects wear volume in the state of adhesive wear. A standard wear test, with ferrous materials using the pin-on-disk method under vertical magnetic field applied by permanent magnets or electro-magnets, has been performed. The wear volume in ferromagnetic materials decreased under a magnetic field. This is expected to be related to that oxidation-promoting effect [5-7], hardness of frictional surface material [6], birth and death of wear particles at friction interface $[7,9]$, protection effect of oxidation film $[7,8]$ and acceleration of severe-mild wear transition [5]. Hiratsuka et al. [5], however, reported that wear volume increased under the horizontal magnetic field. Kumagai et al. [9] examined that wear volume decreases with decreasing magnetic field levels in both states, both sliding wear and reciprocating wear. The result shows that the magnetic effect in reciprocating wear is larger than that in sliding wear. The difference results from different behaviour in discharge of debris, or wear particles in wear process.

\subsubsection{Combination of materials}

In the magnetic field, material combination affects on wear volume that appears increase and decrease. For example, ferromagnetic materials become the mildwear state and wear volume decreases. However, wear of one side may also increase a ferromagnetic material $[10,11]$. This is associated to frictional surface, difference of the hardness, and embrittlement of the wear particles. On the one 
hand, the combination of paramagnetic and diamagnetic is not seen in the most of magnetic effects [12].

\subsubsection{Effect of atmosphere}

As wear volume increases under magnetic field which promotes oxidation, the wear behaviour in vacuum and in inert gases is interested. Kumagai et al. [13] performed experiments changing atmosphere pressure in a range of $10^{5}-10^{0} \mathrm{~Pa}$. The magnetic field decreases wear volume in $\mathrm{Ni} / \mathrm{S} 45 \mathrm{C}$, but an inflection point where wear changes from increase to decrease exists at a pressure of $10^{3} \mathrm{~Pa}$ in $\mathrm{S} 45 \mathrm{C} / \mathrm{S} 45 \mathrm{C}$ : wear increased at pressure less than $10^{3} \mathrm{~Pa}$ and decreased at pressure greater than $10^{3} \mathrm{~Pa}$. Zaidi et al. [6] also reports that wear of steel increases under magnetic field at low pressure of $5 \times 10^{-5} \mathrm{~Pa}$. It is reported for inert gas atmosphere that wear increased [14], and decreased [5] depending on material combination.

\section{Effects of magnetic field on fretting wear}

It has been reported by one of the authors that the wear volume of fretting wear is affected by the magnetic field $[15,16]$ and it increases under some cases of magnetic fields and decreases under other cases. As pointed out in the thesis of J.A. Collins, there are 55-influence factors in fretting, the fretting damage is very complicated. However, the influence of the magnetic field has not been clarified, and then the authors have worked to evaluate the effects by experiments.

\subsection{Experimental details}

In our research, the Herz contact between a ball and a plane has been applied. The detail of the experimental setup was introduced in our previous paper [17]. The fretting wear test device used is shown in Fig.1 (a). The small amplitude of repeated slip motion, at constant amplitude and frequency of sinusoidal wave, was applied using a piezo-electric actuator. The test specimens of SUJ2 steel ball $(19.05 \mathrm{~mm}$ dia. $)$ and SPCC steel plate $\left(\mathrm{W} 10 \times \mathrm{L} 10 \times \mathrm{T} 0.4 \mathrm{~mm}^{3}\right)$ were used. The surface roughness of steel ball and steel plate was $R_{\max }=0.25 \mu \mathrm{m}$ and $0.15 \mu \mathrm{m}$, respectively. Vertical load was applied by dead weight. The vertical load in magnetic fields was applied by considering the value of magnetic attractive force between a ball and a plate. The tangential force was measured by a load cell using strain gage.

The magnetic field was applied to the vertical direction for a contact surface by electromagnet, as shown in Fig.1 (b). Magnetic field experiments were performed using an electromagnet at D.C. current of $1 \mathrm{~A}$ or $3 \mathrm{~A}$. The magnetic density of near the contact surface was calculated using FEM analysis. The results of the analysis show the magnetic density of $B=0.08 \mathrm{~T}$ and $0.22 \mathrm{~T}$, respectively. The worn volume for fretting scar is modelled and evaluated geometrically. The diameter and depth of fretting annulus of the plate were measured with a laser microscope and calculated, as shown in Table 1. 
The tests were carried out without lubricants, at a room temperature of $20-25^{\circ} \mathrm{C}$, relative humidity of $70-80 \%$, and in atmosphere for with and without magnetic field.

Table 1: $\quad$ Test conditions.

\begin{tabular}{c|c|c|c|c}
\hline $\begin{array}{c}\text { Magnetic } \\
\text { density } \\
B[\mathrm{~T}]\end{array}$ & $\begin{array}{c}\text { Applied } \\
\text { load } \\
P[\mathrm{~N}]\end{array}$ & $\begin{array}{c}\text { Fretting slip } \\
\text { range } D[\mu \mathrm{m}]\end{array}$ & $\begin{array}{c}\text { Frequency } \\
f[\mathrm{~Hz}]\end{array}$ & $\begin{array}{c}\text { Number of } \\
\text { cycle } N\end{array}$ \\
\hline $\begin{array}{c}0.08 \text { and } \\
0.22\end{array}$ & $\begin{array}{c}7,20 \text { and } \\
46\end{array}$ & $6-38$ & $\begin{array}{c}10,50,100, \\
150\end{array}$ & $10^{5}-2 \times 10^{6}$ \\
\hline
\end{tabular}

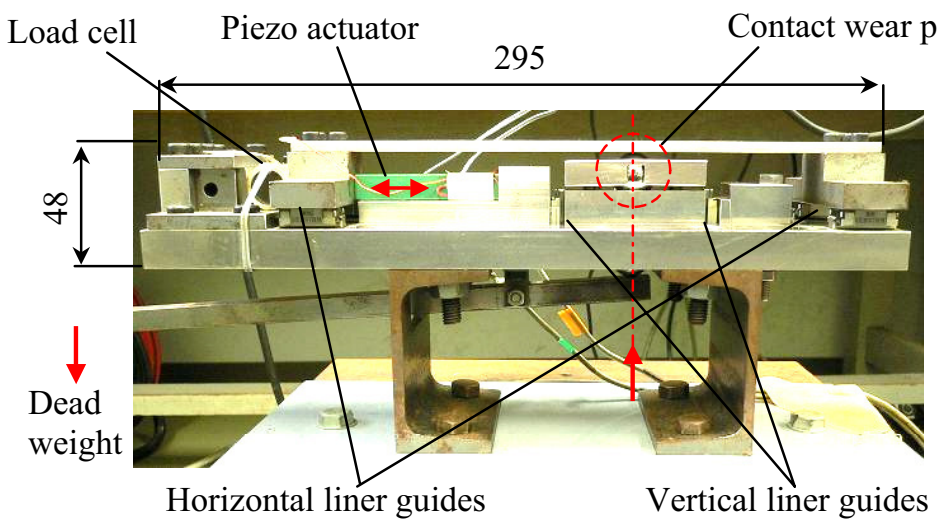

(a) Fretting wear test device used

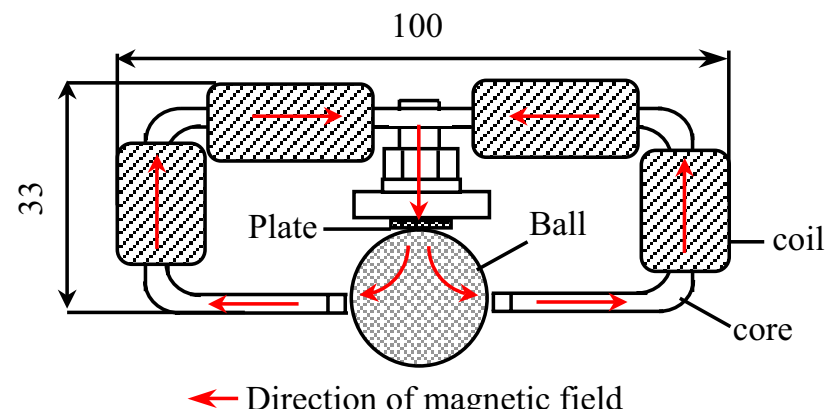

(b) Closed up view of electro magnet used for tests under magnetic field

Figure 1: $\quad$ Fretting wear test rig used.

\subsection{Experimental results}

\subsubsection{Effect of frequency}

The appearance of worn surfaces fretted under different magnetic field densities is shown in Fig.2. The fretted annulus was spread in the case of (a) that is in lower frequency. The fretted annulus is broader at the low frequency of $10 \mathrm{~Hz}$, 
(a)

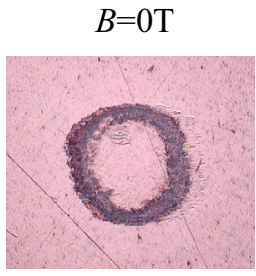

$B=0.08 \mathrm{~T}$

$B=0.22 \mathrm{~T}$

(b)
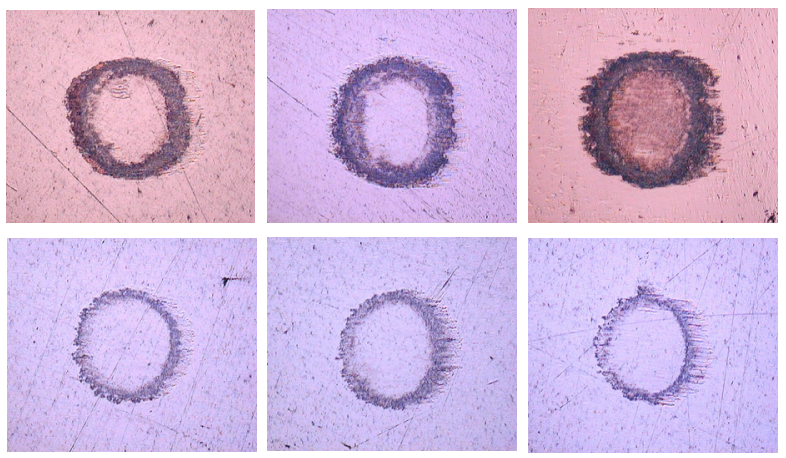

Slip direction

$100 \mu \mathrm{m}$

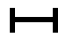

Figure 2: $\quad$ Effects of magnetic field and frequency of vibration on fretting wear annulus with and without magnetic field $(P=20 \mathrm{~N}, D=32 \mu \mathrm{m}$, $N=10^{5}$ ) (a) $f=10 \mathrm{~Hz}$, (b) $f=150 \mathrm{~Hz}$.

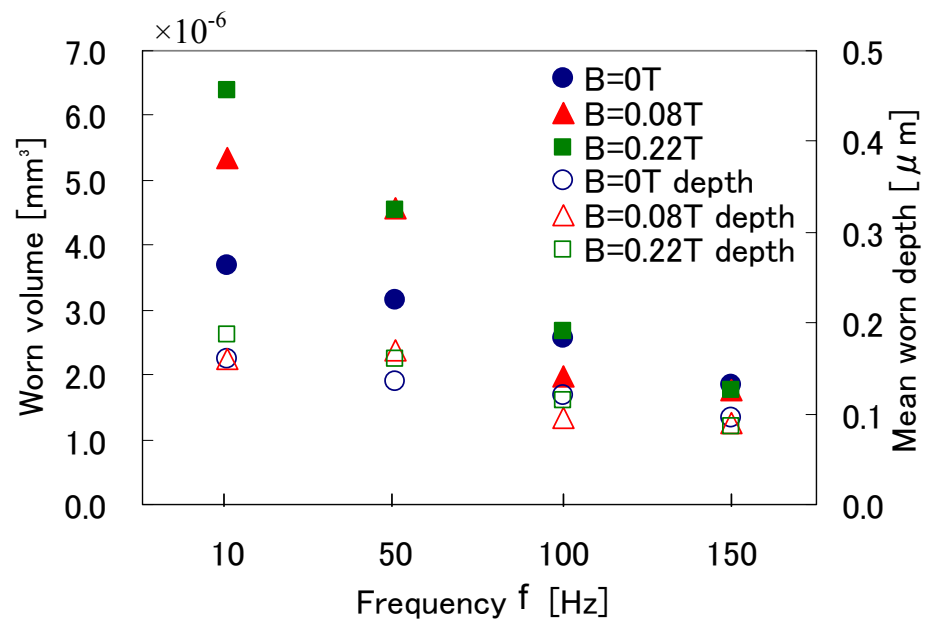

Figure 3: Effect of frequency on worn volume and depth $(P=20 \mathrm{~N}, D=32 \mu \mathrm{m}$, $\left.f=10 \mathrm{~Hz}, N=10^{5}\right)$.

compared with at the high frequency of $150 \mathrm{~Hz}$. The mean outer diameter of the annulus and worn volume are shown in Fig.3. The latter decreased with increasing frequency, and increased with increasing magnetic field density. That is due to mainly time dependency based on oxidation effects regarding to magnetic flux distribution in the contact area [16].

\subsubsection{Effect of applied load}

The slip motion is restrained by higher applied load, and then the state of contact becomes the Stick-Slip behavior. As a result, the influence of the magnetic field is decreased in the Stick-Slip region. At the high cycle region around $10^{6}$ or 


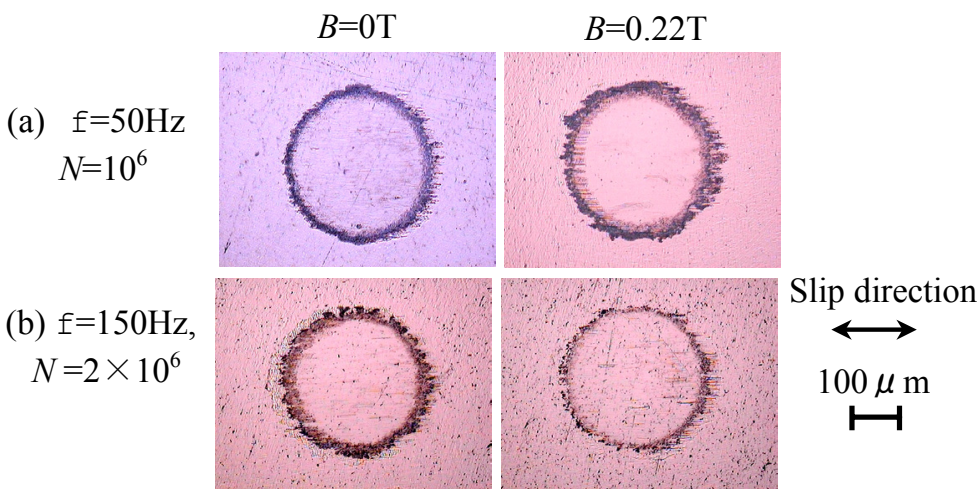

Figure 4: Effects of magnetic field and frequency of vibration on fretting wear annulus with and without magnetic field $(P=46 \mathrm{~N}, D=32 \mu \mathrm{m})$.

$2 \times 106$ cycles, as shown in Fig.4. However, the influence of the magnetic field appeared similar to that at the lower applied load.

\subsubsection{Effect of slip amplitude}

The experimental result shows the effect of slip amplitude as follows: the wear morphology differs among Stick: Stick-Slip, and Slip regions, the increase in slip value is to clear form wear annulus and to be severe: the slip amplitude varied from the Stick-Slip region to the Slip region in the range of $32-38 \mu \mathrm{m}$, and increases the discharge of wear particles.

Under the magnetic field, fretting scar changed when slip amplitude is large. The magnitude of slip amplitude appears in the change in the fretting scar. Fig.5 shows the relationship between two factors of $b / a$ and $Q / P$, where $b / a$ is the ratio of the fretting annulus diameter: $b / a=1$ is the Stick state, $b / a=0$ is the Slip state, and $0<b / a<1$ is the Stick-Slip state. Experiment results are also plotted in Fig.5. Under the magnetic field, the values of $b / a$ decrease with increase in $Q / P$. The decrease in the value of $b / a$ in the magnetic field is greater in the range of $Q / P=0.5-0.6$, that is, the Stick-Slip region. The Stick region accelerates wear damage under the magnetic field (Fig.6). By the way, wear particles generated at the Slip region in the magnetic field are trapped, and therefore the worn volume is increased by the acts of abrasive particles which piled-up at the contact surface neighbourhood.

\subsection{Accelerated oxidation under magnetic field}

The worn volume is decreased by decompression, as results show in Fig.7 and the worn volume decrease to about $1 / 4$. In vacuum $(24 \mathrm{~Pa})$, worn volume does not change with and without magnetic field. In air, in the magnetic field the oxidation of the contact surface and wear particles are accelerated. That is considered to be resulting from reddish brown particles formed under the magnetic field. The authors, then, tried to measure the oxygen ingredient concentration of fretting scar about a contact surface in the slip direction by 


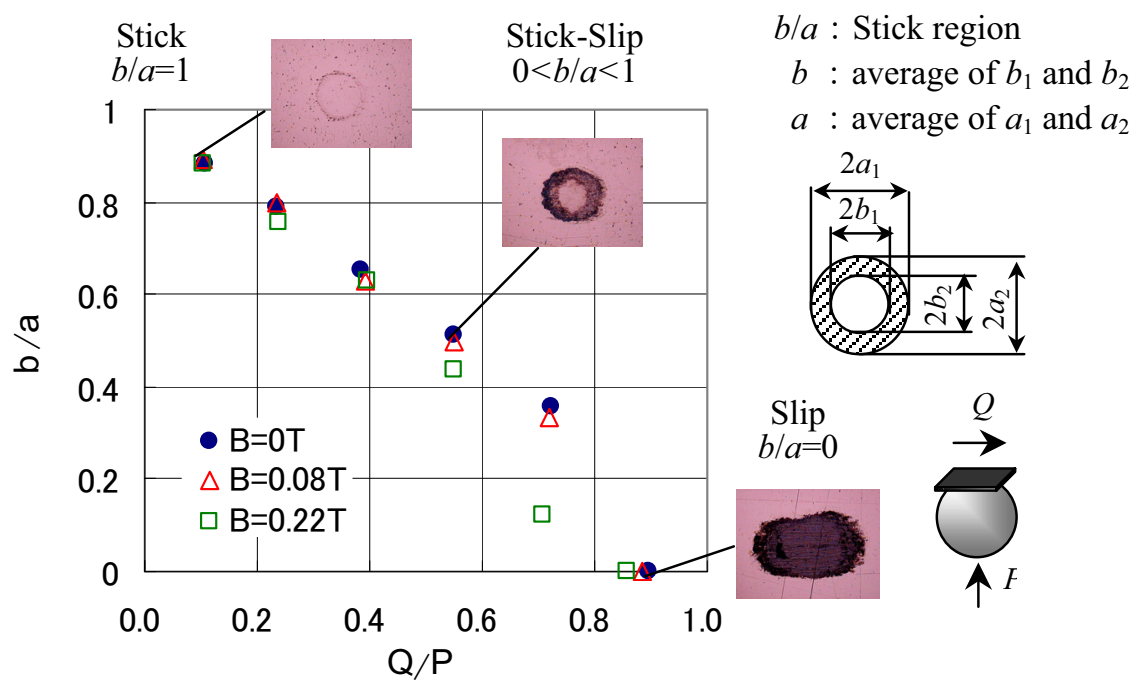

Figure 5: $\quad$ Relation between $Q / P$ and $b / a$.

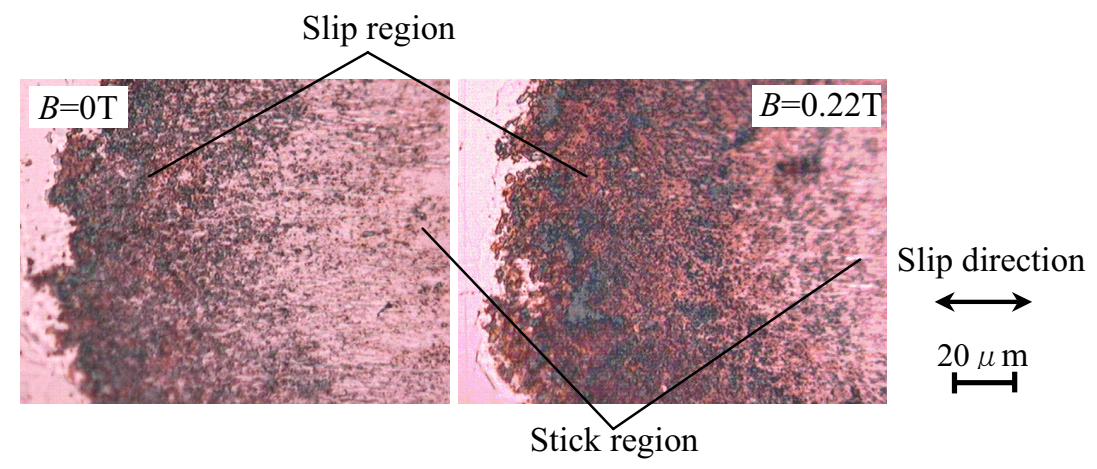

Figure 6: Laser micrographs of fretting wear scars on with and without magnetic field. $(D=32 \mu \mathrm{m})$.

EPMA line analysis. The results are shown in Fig.8. Under the magnetic field, average oxygen concentration of worn scar was increased: In air is by $10 \%$ and in vacuum is $19.5 \%$. In fretting wear process, the magnetic field promotes oxidation and proceeds to convert the reddish brown wear particles from black wear particles. 
70 Tribology and Design
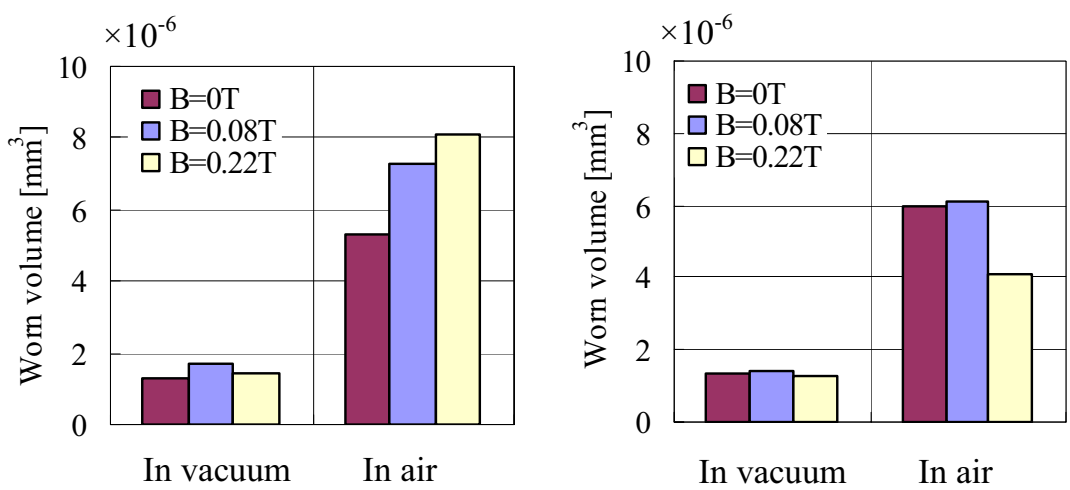

Figure 7: Relation between worn volume and air pressure. (a) $\mathrm{f}=10 \mathrm{~Hz}$, $\mathrm{D}=25 \mu \mathrm{m}, \mathrm{N}=105$; (b) $\mathrm{f}=150 \mathrm{~Hz}, \mathrm{D}=32 \mu \mathrm{m}, \mathrm{N}=105$.
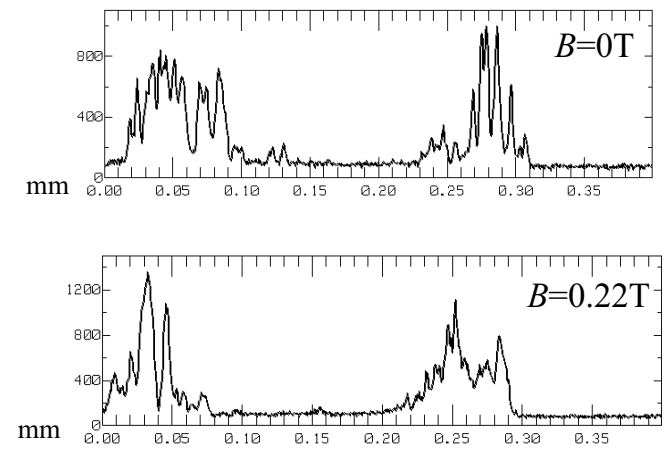

(a) In air.

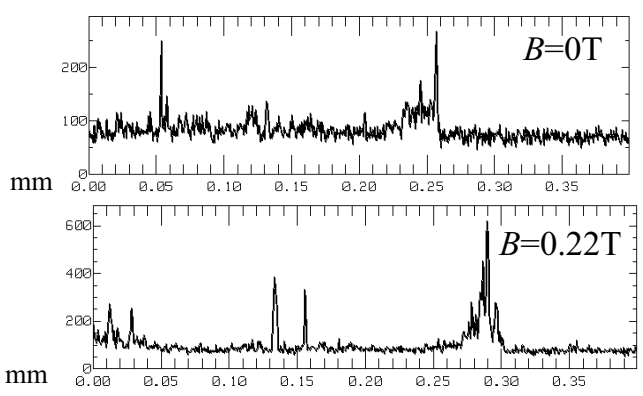

(b) In vacuum.

Figure 8: Oxygen analysis of worn surface by EPMA. ( $P=7 \mathrm{~N}, D=25 \mu \mathrm{m}$, $f=10 \mathrm{~Hz})$. 


\subsection{Fretting map}

The effect of magnetic field on wear morphology can be well understood through the fretting map showing the relationship between frequency and slip amplitude. That is shown in Fig.9. The effect of magnetic fields appeared only a boundary between the Stick-Slip and the Slip regions. The Slip region is reduced to slip amplitude at lower frequency by existence of a magnetic field, and the Stick-Slip region is shifted to high slip amplitude at higher frequency. In a long duration up to $\mathrm{N}=10^{5}$ cycles, oxidization of the wear particles is accelerated in low frequency and they behave an abrasive material and act to decrease the Stick region.

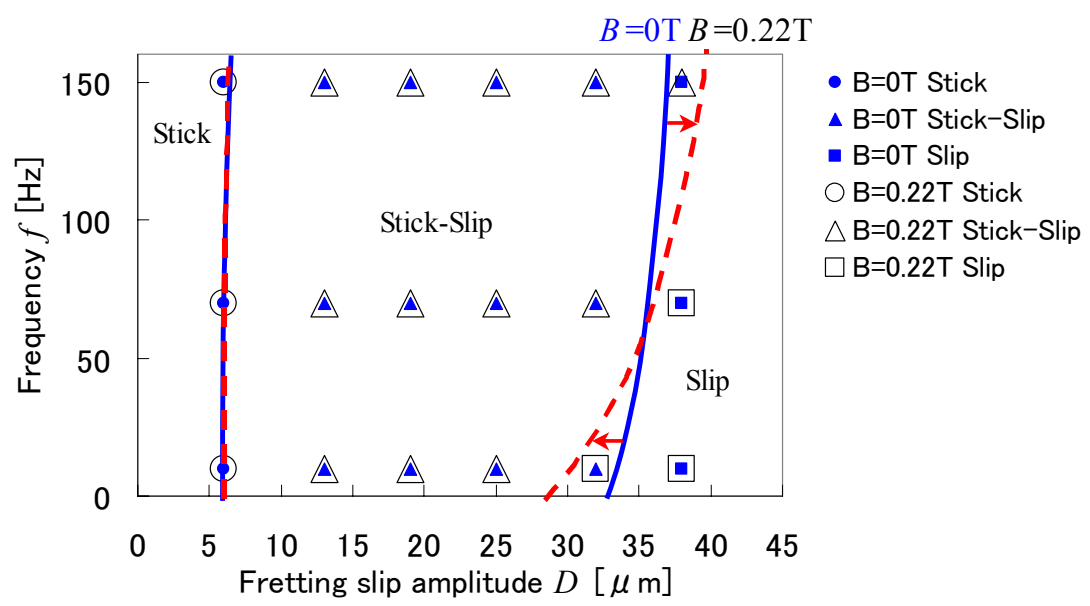

Figure 9: Fretting map with and without magnetic field $\left(P=7 \mathrm{~N}, N=10^{5}\right)$.

\section{Conclusion}

The effects of magnetic fields on machines have to be clarified for working under the magnetic fields environment. The effect of the magnetic field is very complicated; it is also known that there are many reports that the magnetic fields take place with opposite results. The experimental results showed that the wear region spread or narrowed under the magnetic field, as shown in the fretting map. This map is indicating the recommendation for the design of electric apparatus.

On the other hand, the applied technology of the magnetic field is reported. For example, in magnetic polishing in the processing [18], another, in the artificial joint in biotribology, problems such as the toxicity to the human body of the wear particles or the durability occur. Nakanishi et al. [19] applied a magnetic field to give an examination of the wear reduction, and therefore it is 
necessary to consider the influence on the human body and machinery under the magnetic field environment. In addition, with development of the machinery by superconductivity technology, mechanical behaviour of metallic materials under a magnetic field is examined [20]. However, in the superconductivity technology, the magnetic density is rising as shown in Fig.10 [21].

Therefore, we must do more research to clarify the influence of the magnetic field on machine and structures, because high technologies such as superconductivity technologies will be developed more rapidly.

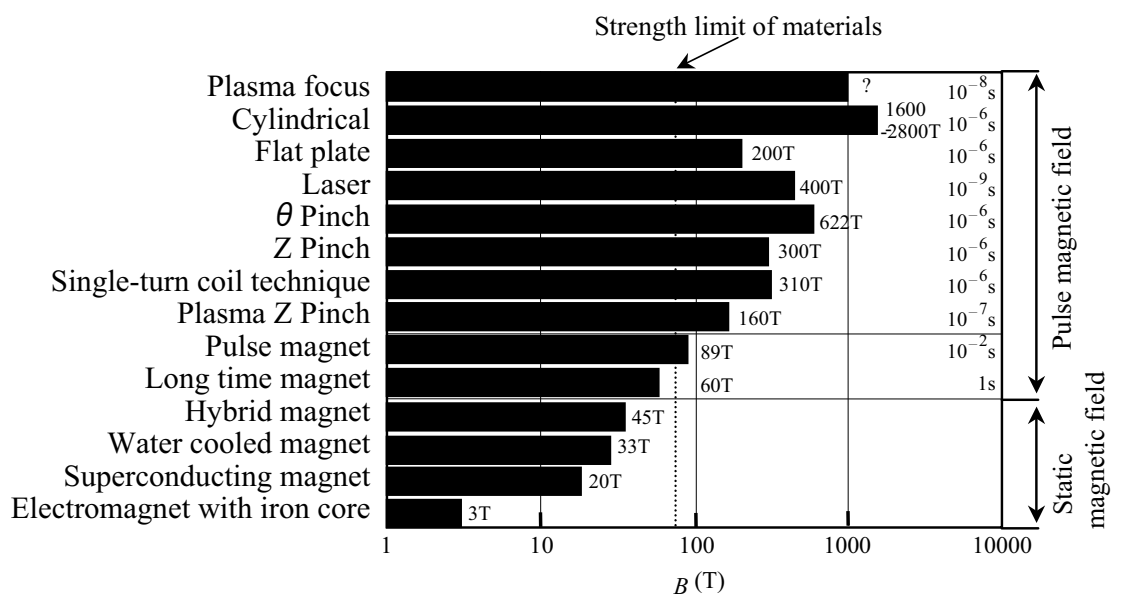

Figure 10: High magnetic field generation method and maximum magnetic field [21].

\section{References}

[1] K.Okada; Journal of Japanese Society of Tribologists, Vol.34, No.5 (1989) 379-380.

[2] A.Sato: Journal of Magnetics Society of Japan, Vol.21, No.4-2 (1997) 753756.

[3] A.Celik, A. F.Yetim, A.Alsaran and M.Karakan, Materials and Design 26 (2005) 700-704.

[4] M.K.Muju and A.Ghosh, Wear, 41 (1977) 103-116.

[5] K.Hiratsuka, T.Sasada and S.Norose; Wear 110 (1986) 251-261.

[6] H.Zaidi, M.Amirat, J.Frene, T.Mathi and D.Paulmier; Wear, Vol.263, Issues 7-12, (2007) 1518-1526.

[7] K.Kumagai, O.Kamiya and S.Sato; Journal of Japanese Society of Tribologists; Vol.36, No.9 (1991) 727-734.

[8] H.Zaidi, K.J.Chin and J.Frene; Surface and Coatings Technology 148 (2001) 241-250.

[9] K.Kumagai, M.Mizui and O.Kamiya; Journal of Japanese Society of Tribologists, Vol.35, No.10 (1990) 741-748. 
[10] M.El Mansori, H.Zaidi, E.K.Kadiri, and D.Paulmier; Surface and Coatings Technology 86-87 (1996) 511-515.

[11] H.zaidi, A.Senouci; Surface and Coatings Technology 120-121 (1999) 653658.

[12] K.Hiratsuka, H.Saito and T.Sasada; Journal of Japanese Society of Tribologists, Vol.34, No.6 (1989) 430-436.

[13] K.Kumagai, H.Kawane and O.Kamiya; Scientific and Technical Reports of the Mining College, Akita University, 16, (1995) 51-58.

[14] M.El.Mansori, M.Schmitt and D.Paulmier; Surface and Coatings Technology 108-109 (1998) 479-483.

[15] K.Sato, T.A.Stolarski and Y.Iida; Wear Vol, 241 (2000) 99-108.

[16] Y.Iida, Y.Nasu and K.Sato; JSME Annual Meeting, Vol.2005, No4. 291292.

[17] Y.Nasu and K.Sato; Transactions of the Japan Society of Mechanical Engineers, Vol.74, No.743, (2008) 1026-1030.

[18] O.Nakano; The Journal of the Surface Finishing Society of Japan, 57(11), (2006) 764-767

[19] Y. Nakanishi, T.Murakami and H. Higaki; Transaction of Japan Society of Mechanical Engineers. C, Vol. 164, No. 626 (1998) 3952-3958.

[20] E,Fukushima; Tetsu to Hagane, The Iron and Steel Institute of Japan, 75(6) (1989) 879-886

[21] N.Miura; Experimental Physical Chemistry series, Vol.5, Generation and Application of High Magnetic Fields (2008) KYORITSU SHUPPAN 